\title{
Experiences of an Ophthalmologist during Evaluation and Motivation of Patients with Low Vision in a Low Vision Aid Cell
}

\author{
Dr. Almas Abdul Aziz ${ }^{1}$, Dr. Uma Kulkarni² \\ ${ }^{1,2}$ Department of Ophthalmology, Yenepoya Medical College, Mangalore
}

\begin{abstract}
Aim: Explore the challenges faced by an ophthalmologist during evaluation and counselling of a patient with low vision. Material and methods: After establishment of the new LVA centre, the challenges faced at various steps in the establishment and running of the $L V$ centre are described under the heading 1) Establishing the LV centre 2) Introducing LVA to the patients 3) Motivating patients with LV for LVA 4) Evaluation using other ancillary tests 5) Dealing with 'no improvement in vision' 6) C ounseling Observation and discussion: Even though setting up of a low vision centre was not difficult, the patients' awareness about such services was poor due to the 'weird appearance' and 'closer working distance' of these LVAs. Patients had more preference for spectacles, medical or surgical management for their low vision rather than LVAs. Most patients were poorly motivated, and manifested a ' given up' attitude probably as a result of psychological impact of low vision. Performing ancillary visual tests was found to be more time consuming and added no value to the result. It was also difficult to explain to some patients why they did not improve with low vision aids which may worsen their already depressed state of mind. The authors felt the need for a trained counsellor for motivation and counselling of patients with low vision, more so in cases where there was futility of treatment and perceived lack of family support. Conclusion: Active and sustained efforts are required in motivating the LV patients for LVA. They should be holistically managed with contributions from counsellor, psychiatrist or psychologists with equal contribution from the patient and the care takers.
\end{abstract}

Keywords: LVA, vision, spectacles, motivation, counselling

\section{Introduction}

Low vision (LV) is a form of visual impairment which is not amenable to treatment with routine optical, medical or surgical means. It impacts the quality of life of the individual through its effect on profession, social and leisure activities and also personal care. It adds to the societal burden of visual impairment.

However, many of the individuals with LV do have some residual vision which can be effectively utilised, through the use of low vision aids (LVA). But centres with LVA services are functional only in the bigger eye care centre in India ${ }^{[1]}$ and there is a need to widely distribute the $\mathrm{LV}$ centres. This article puts light on the experiences faced while providing LVA in a newly established low vision centre.

\section{Review of Literature}

The prevalence of visual impairment is increasing in parallel with increasing longevity. At least one in a hundred south Indian populations has low vision ${ }^{[2]}$. The causes for low vision in India include retinal diseases, amblyopia, optic atrophy, glaucoma and corneal diseases ${ }^{[3]}$. In the younger age group, myopic macular degeneration and optic neuropathy are important contributors to the poor vision ${ }^{[4]}$.

In people with low vision, LVAs permit practical adaptations for daily living tasks ${ }^{[1]}$ improving their quality of living. However, the rate of utilisation of low vision aids is not commensurate with the prevalence of low vision. The chief barriers included lack of awareness about low vision rehabilitation and poor expectations form LVAs ${ }^{[5,6]}$. The effective functioning of a LV centre depends on overcoming the barriers for utilisation of LVAs.
Most of the studies regarding barriers for utilization of LVAs are based on questionnaire and awareness studies. Our study describes the experiences of the ophthalmologist during evaluation and counselling of a patient with low vision who is referred for the prescription of LVA. The study identifies barriers faced by the ophthalmologist in effectively communicating the usefulness and failures of LVAs among patients with low vision.

\section{Aim:}

The aim of our study was to understand the challenges faced by an ophthalmologist during evaluation and counselling of a patient with low vision.

\section{Material and methods:}

50 participants with low vision were enrolled for studying the patterns of LVA prescription and utility after obtaining the Institutional Ethics Committee and after administering informed consent from the participants. This article discusses the challenges experienced by the investigator during the evaluation and counselling of patients with low vision. The confidentiality of the participants, based on whose reactions, the experiences of the investigator have been narrated in this article are kept confidential.

With the increasing number of cases of low vision noted in the institute, a LV centre was established in the ophthalmology department of the medical college in the year 2014. The centre was established with a set of optical LVA, procured from different dealers in India. The basic kit procured for the establishment of the low vision centre included: 


\section{International Journal of Science and Research (IJSR) \\ ISSN (Online): 2319-7064 \\ Index Copernicus Value (2013): 6.14 | Impact Factor (2014): 5.611}

1) The diagnostic tools:

a) 3 meter LogMar chart of the ,I-test ${ }^{e e}$ for distant visual acuity

b) Standard pin hole for pin hole testing

c) Near vision flip charts for testing near vision at a working distance of $25-30 \mathrm{~cm}$.

d) Contrast sensitivity testing at 3 meter distance

e) Amsler grid and Humphrey Field analyser for testing central visual field

f) Worth Four dot test for testing binocular vision

2) Low vision aids for near vision:

a) Standard hand held (+6D, +14D and +20D),

b) Stand mounted (+8D, +14D and +20D)

c) Spectacle magnifiers (+6D, +10D, +16D, +20D)

3) Low vision aids for distant vision:

a) $2.5 \times$ magnifier telescope

b) Clip- on telescope

A protocol was followed for evaluation and prescription of LVAs for patients with low vision referred from specialty clinics of the department after a detailed clinical evaluation. The difficulties faced at various steps in the establishment and running of the $\mathrm{LV}$ centre were noted and classified for discussion into the following categories.

1) Establishing the LV centre

2) Introducing LVA to the patients

3) Motivating patients with LV for LVA

4) Evaluation using other ancillary tests

5) Dealing with, no improvement in vision

6) Counseling

\section{Observations and Discussion}

The observations are given and discussed under the following headings:

1) Establishing LV center: The establishment of a low vision center did not constitute any perceived barrier. It was possible to establish the centre in the available space and little investment for the low vision trial set. Equipment required for the evaluation of patients was already available in the specialty clinics. Hence starting a low vision centre was not difficult in institutions like medical colleges.

2) Introducing LVA to the patients: Only 10 out of the 50 cases of low vision were aware of ,low vision devices $^{\text {ee }}$ In the backdrop of poor awareness, it was a challenge for the ophthalmologist to introduce the concept of ,special lenses or low vision devices ${ }^{\text {ee }}$ to the remaining $80 \%$ of the cases. A great resistance was perceived during counseling a patient for LVA. When the only possible option for the management of low vision was LVA, which most patients were not aware, the challenge was in dealing with their perceptions of strangeness and novelty of the LVA, particularly with the stand-held or hand-held magnifiers for near and the telescopes for distance. Some patients described the LV, especially the telescopes to be 'weird appearing' and did not choose to try it. For those who improved with the LVA for near, weren 't so happy because of the close working distance as if ,holding it against the nose'. It was observed during the interactions with LV patients, that patients are more inclined towards accepting traditional methods of management, namely, spectacles, eye drops or surgery. The probable reasons for better acceptance of other modalities of management are:

a) Spectacles: Spectacles are well-known to patients from several decades in the management of diminution of vision for most, if not all ophthalmic conditions, either as a prime modality or as a supplement to surgical or medical management. Prescribing spectacles is not a novel method and is easily acceptable in the present day by most patients. Challenges have been explored, however in the acceptance of spectacles among patients, mostly for cosmetic concerns ${ }^{[7]}$. For conditions where vision cannot be improved with spectacles, the general perception of the patients is ,If spectacles are not beneficial in improving vision, then there is a need for surgical or medical management ${ }^{\text {ec }}$.

b)Medical management: Medical management of ocular ailments is easily acceptable to patients since they form the mainstay of management of common ophthalmic conditions and is well-known and easy to administer. Although, challenges have been explored in long term compliance, chronic conditions like glaucoma and while dealing with adverse effects ${ }^{[8]}$. Many patients are known to buy eye drops from medical shops without prescriptions.

c) Surgical management: Convincing a patient for surgical management for conditions like cataract, glaucoma and retinal disorders is mostly straightforward for a motivated and educated patient. It may pose difficulties in some patients, who are either not motivated ${ }^{[9]}$ or in whom the visual prognosis has been predicted to be poor. However, ocular surgery is well known and generally acceptable to patients.

d)Others: With the increasing screening of diabetics for retinopathy and the early treatment of Diabetic retinopathy with lasers and intra-vitreal injections, patients are gradually becoming aware of these newer modalities of management. There are no studies to know the acceptance of these methods, but it is perceived to be a hopeful method of management. Some patients are even interested in knowing, whether eye transplantation can be done to improve vision". Young patients with low vision have even enquired about ,computer chip in the eye referable to the 'bionic eyee $e^{\text {ee }}$ However, this was only observed among the young and educated patients.

3) Challenges in motivating patients for LVAs: Another challenge was in convincing patients who came to the LV centre after having lost hopes of gaining back vision with all routine methods of management. Many patients ,give upe due to failure of improvement in vision by other modalities of management tried at different centres and by different ophthalmologists, over the past several years. „Loss of hope of gaining back vision ${ }^{\text {ee }}$ is known to cause ,depression ${ }^{\text {ee }}$ [9] and suicidal tendencies among patients with blindness. Several socio-economic factors like, dependence on others, rejection by family and financial burden add to the complexity. Most patients suffer in multiple dimensions owing to blindness and hence are not so willing to take up any more options positively ${ }^{[10]}$. One of the patients with $L V$ improved with LVA but was not motivated enough to buy it. He rather asked us to 'certify him as blind, so that he could get the 


\section{International Journal of Science and Research (IJSR) \\ ISSN (Online): 2319-7064}

Index Copernicus Value (2013): 6.14 | Impact Factor (2014): 5.611

government benefits'. This reflects the ,give up ${ }^{\text {ce }}$ attitude of the blind, which may again be a psychological manifestation of blindness. Hence it is important for the ophthalmologist to keep in mind that introducing the concept of ,low vision aids ${ }^{\text {ee }}$ as a means of improving vision poses challenges, in the first instance, owing to an unmotivated patient.

4) Evaluation using other ancillary tests: The low vision evaluation includes vision testing for distance and near, anterior and posterior segment examination of the eye with slit lamp and ophthalmoscopy. Apart from these tests, many other ancillary tests for visual function were included in the protocol like contrast sensitivity, colour vision, worth 4 dot test and Amsler grid. It was found that when vision was very poor, subjecting them to these ancillary tests added no further information, owing to the fact that most of the patients could not even visualise the test charts. The tests consumed more time (at least another 30-40 minutes) without adding any additional value in diagnosis or management in most patients. This tested the patience of the patients who were already ,not motivated $^{\text {ee }}$ to the new and strange method of management. Further, it demoralised many patients. In some patients, additional sessions were needed for such ancillary tests.

5) Dealing with 'no improvement' in vision with LVAs: About two-thirds of the patients did not show improvement in vision with LVA. It was a challenge to handle patients who showed, no improvement ${ }^{\text {ee }}$ in vision with any of the low vision aids. The train of events including the ,diagnosis ${ }^{\text {ee }}$ of blindness, the ,futility ${ }^{\text {ee }}$ of management options, the longs hours of evaluation time, the cross-references between departments, the need for by-standers and support for mobility, and then the motivation and hopefulness at the LV centre followed by the statement, no further improvement ${ }^{\text {ec }}$ were enough to depress the person. It was difficult for the patients to understand why the ,special lenses ${ }^{e e}$ for which they were motivated ,did not work ${ }^{e c}$ on them. It was also depressing for the ophthalmologist to send the patients back without improvement in vision. It is important to keep in mind that such patients may face a nervous breakdown and need for psychiatric counseling ${ }^{[11]}$. The doctor-patient relationship in such instances is strained despite efforts from the doctor to give the best to the patient. It was a disheartening to hear a patient (who did not improve even with the highest power of LVA) who said "is it possible to use your research and give me extremely high powered lens so that I can see". It is this patient who has visited the LV centre at least five times each time, wanting to try more LVA and continues to come.

6) Counseling: The most difficult challenge after the establishment of LV centre in the hospital was counseling the patient in case of failure of LVAs in improving vision. The authors feel the need of trained personnel for counseling the patients in such circumstances. The need for counselor was also felt in cases where family support was lacking when it came to purchasing the LVA.

\section{Conclusion}

Our experience during the evaluation of about 50 patients with low vision over the period of 18 months indicates that there are more aspects of the patients to be addressed rather than only visual parameters. The challenges faced by the ophthalmologist are located more at the psychological level from the patient's point of view, rather than at the ocular level. The challenges are also located at the level of communication skills of the ophthalmologist, rather than just clinical skills. Efforts are required for transcending the usual beliefs and acceptances of the patients, dealing with futility of treatment associated with the last options for management of visual impairment. There is a need for more efficient and extensive counselling, which is not routine part of training of ophthalmologists. Another key factor could be spending sufficient time on trial with multiple sessions. It requires a lot a practice and time to imbibe the skills of empathy and professionalism apart from competency in management of such cases. It may not be an overemphasis to state that patients with low vision should be holistically managed with contributions from counsellor, psychiatrist or psychologists with no less contribution from the patient and the care takers.

\section{References}

[1] Are the low vision devices available in India? " DoctorNDTV Queries [Internet]. Doctor.ndtv.com. 2003 [cited 15 August 2013]. Available from: http://doctor.ndtv.com/faq/ndtv/fid/2662/Are_the_low_visi on_devices_available_in_India.html

[2] Rakhi $D$ et al, planning for low vision in India Ophthalmology Volume 109, Issue 10, pages 1871-1878, October 2002

[3] Nakamura Y, Tomidokoro A, Sawaguchi S, Sakai H, Iwase A, Araie M, Prevalence and causes of low vision and blindness in a rural Southwest Island of Japan: the Kumejima study. Ophthalmology. 2010 Dec;117(12):231521.

[4] Congdon N. Important Causes of Visual Impairment in the World Today. JAMA. 2003;290(15):2057.

[5] Van Boemel G.B: Barriers to low vision care: why some visually never learn technologies available to them

[6] Pollard $\mathrm{T}$ et al: barriers to accessing low vision services. Ophthal. physiol. opt (2003);23:321-327

[7] Savur S. The Perceptions Regarding Refractive Errors and Their Psychosocial Impact on Youth in Dakshina Kannada. Journal of Clinical and Diagnostic Research. 2011;5(4):746-748.

[8] Bansal R, Tsai J. Medical Treatment: Compliance/Adherence to Glaucoma Medications-A Challenge. Current Journal of Glaucoma Practice with DVD. 2007;1(2):22-25.

[9] Shetty R, Kulkarni UD. Change-readiness of the blind:A hospital based study in a coastal town of South India. Middle East Afr J Ophthalmol 2014;21:158-64

[10] Lam N, Leat S. Barriers to accessing low-vision care: the patient's perspective. Canadian Journal of Ophthalmology / Journal Canadien d'Ophtalmologie. 2013;48(6):458-462.

[11] Evans r. Loneliness, depression, and social activity after determination of legal blindness. Psychological Reports. 1983;52(2):603-608. 